\title{
ERRATUM \\ Three-dimensional instabilities over a rectangular open cavity: from linear stability analysis to experimentation - ERRATUM
}

\author{
J. de Vicente, J. Basley, F. Meseguer-Garrido, J. Soria and V. Theofilis
}

doi:10.1017/jfm.2014.126, Published by Cambridge University Press, 28 April 2014

Key words: cavity flow, instability, erratum

In de Vicente, Basley, Meseguer-Garrido, Soria \& Theofilis (2014) an incorrect file for figure 18(a) was published. The publisher apologises to the authors and readers for this error. The correct figure is shown on the following page.

\section{REFERENCE}

de Vicente, J., Basley, J., Meseguer-Garrido, F., Soria, J. \& Theofilis, V. 2014 Three-dimensional instabilities over a rectangular open cavity: from linear stability analysis to experimentation. J. Fluid Mech. 748, 189-220. 


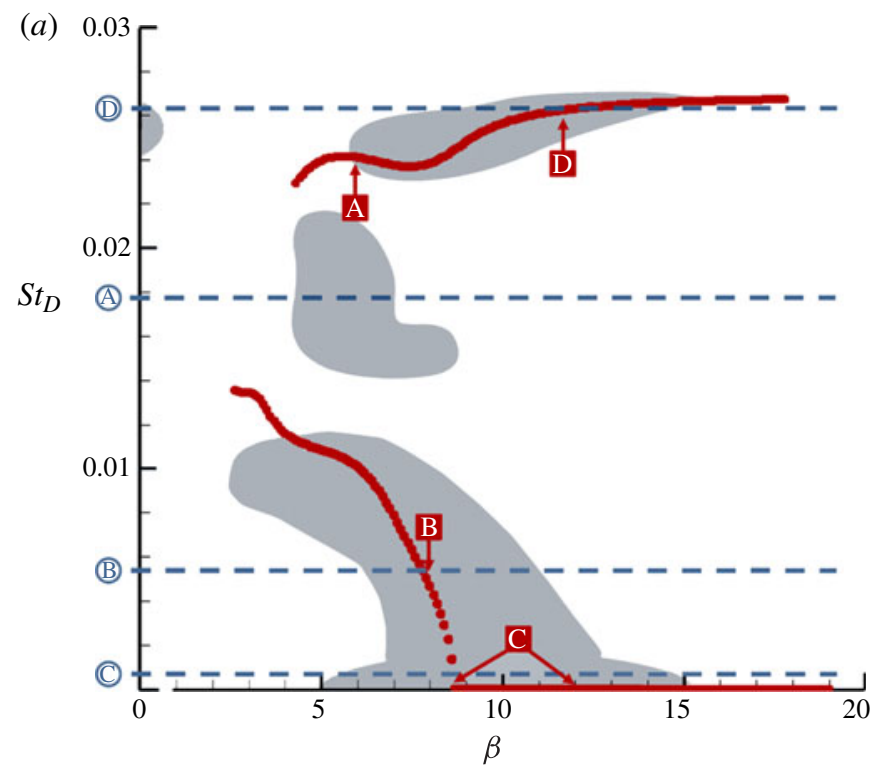

(b)

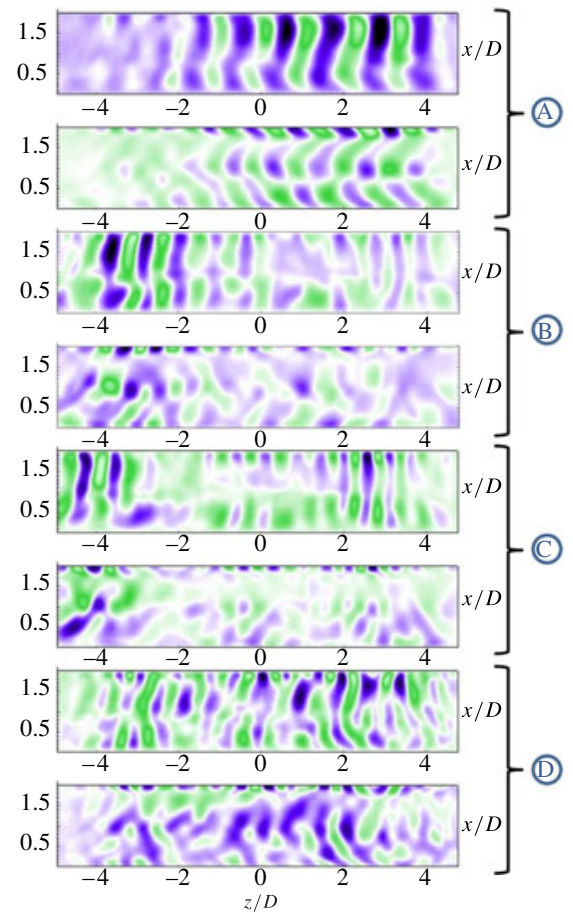

(c)
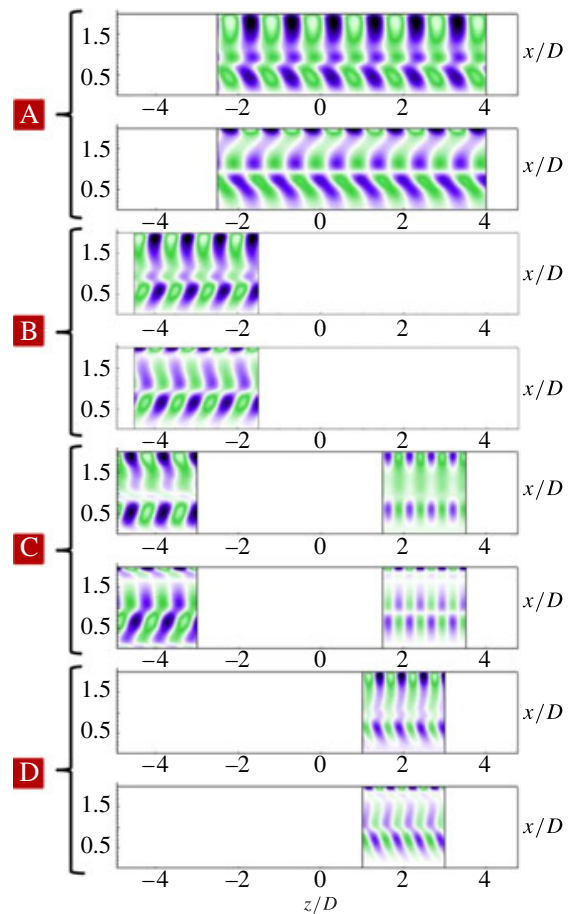

Figure 18. (Colour online) Main flow features at $R e_{D}=2400$ for both experimental and linear stability analysis. (a) BiGlobal unstable eigenvalues (dots) and qualitative schematic depiction of the most energetic modes in the experiments (shades) in the ( $\beta$, $S t_{D}$ )-plane. $(b, c)$ Velocity fields related to the four representative modes highlighted in $(a)$ : (b) corresponds to global Fourier modes from the experimental dataset; $(c)$ presents the reconstructed flow using BiGlobal analysis. For each mode, streamwise velocity (top plot) and spanwise velocity (bottom plot) are shown. 